\title{
Prevalence and mortality of cardiovascular events in patients with hyperthyroidism: a nationwide cohort study in the United States
}

\author{
Mohammad Hussein ${ }^{1}$, Eman Toraih ${ }^{1,2} \wedge$, Adin S. C. Reisner ${ }^{3}$, Peter Miller ${ }^{3}$, Ralph Corsetti ${ }^{1}$, Emad Kandil ${ }^{1}$ \\ ${ }^{1}$ Department of Surgery, Tulane University, School of Medicine, New Orleans, LA, USA; ${ }^{2}$ Genetics Unit, Department of Histology and Cell Biology, \\ Faculty of Medicine, Suez Canal University, Ismailia, Egypt; ${ }^{3}$ Tulane University, School of Medicine, New Orleans, LA, USA \\ Contributions: (I) Conception and design: M Hussein, E Toraih, E Kandil; (II) Administrative support: M Hussein, E Toraih, E Kandil; (III) Provision \\ of study materials or patients: All authors; (IV) Collection and assembly of data: M Hussein, E Toraih; (V) Data analysis and interpretation: All \\ authors; (VI) Manuscript writing: All authors; (VII) Final approval of manuscript: All authors. \\ Correspondence to: Prof. Emad Kandil, MD, MBA, FACS, FACE. Department of Surgery, Tulane University School of Medicine, New Orleans, LA, \\ USA. Email: ekandil@tulane.edu.
}

Background: Little is known about the implications of hyperthyroidism on cardiovascular diseases like myocardial infarctions (MI), ischemic strokes, and hemorrhagic strokes. Previous studies implicate hyperthyroidism as a risk factor for MI and ischemic stroke. Cardiovascular disease is the leading cause of death in the US, and the possibility of hyperthyroidism contributing to this disease burden warrants investigation in a US patient population.

Methods: The National Readmission Database (NRD) from 2010 to 2017 was used to identify adults who had a new-onset diagnosis of MI, ischemic stroke, or hemorrhagic stroke. Risk factors for mortality, prolonged hospital stay, and hospital cost were analyzed with binary logistic regression.

Results: A total of 278,609,748 hospital records were reviewed. Of these, 15,685,461 were hospitalized for adverse cardiovascular events with concomitant hyperthyroidism, roughly 4.5 per 1,000 cases. Patients with $\mathrm{MI}$ and hemorrhagic stroke with thyrotoxicosis had a lower mortality rate than euthyroid patients (OR 0.81, 95\% CI: 0.78-0.84, $\mathrm{P}<0.001$ and OR 0.72, 95\% CI: 0.67-0.77, $\mathrm{P}<0.001$, respectively). Thyrotoxicosis was associated with increased hospital stay (MI: OR 1.35, 95\% CI: 1.32-1.38, P<0.001; ischemic: OR 1.38, 95\% CI: 1.35-1.41, $\mathrm{P}<0.001$; hemorrhagic: OR 1.33, 95\% CI: 1.25-1.40, $\mathrm{P}<0.001)$, expenditures in ischemic (OR 1.31, 95\% CI: 1.28-1.34, $\mathrm{P}<0.001$ ) and hemorrhagic stroke patients (OR 1.18, 95\% CI: 1.12-1.24, $\mathrm{P}<0.001$ ).

Conclusions: Patients that experienced MI or hemorrhagic stroke with concomitant thyrotoxicosis were observed to have a lower mortality rate than euthyroid patients but had with an increased length of hospital stay. Patients who had ischemic or hemorrhagic stroke with thyrotoxicosis had a significantly higher hospital cost. The hyperdynamic state underlying hyperthyroidism may be protective in these adverse events. Further studies into these physiological changes are warranted.

Keywords: National database; thyrotoxicosis; myocardial infarctions (MI); ischemic strokes; hemorrhagic strokes

Submitted Apr 10, 2021. Accepted for publication Jul 22, 2021.

doi: 10.21037 /gs-21-236

View this article at: https://dx.doi.org/10.21037/gs-21-236

^ ORCID: 0000-0001-8278-7094. 


\section{Introduction}

Cardiovascular disease is the leading cause of death in the United States (US) and accounts for nearly 200 billion dollars in health expenditures, not including lost job productivity (1). The morbidity, mortality, and financial costs of heart disease are projected to increase over the coming decade. Prevalence of all cardiovascular disease is projected to rise from the 2010 level of $36.9 \%$ to $40.5 \%$ by 2030 , with associated direct health care costs projected to triple over that timeframe (2). Projections of unsustainable healthcare expenditure growth are a national concern and the Congressional Budget Office has projected outlays from major healthcare programs to rise from $5.2 \%$ to $9.3 \%$ of national GDP from 2019 to 2049 should laws remain unchanged (3). The scope of the financial and health costs associated with cardiovascular disease makes an investigation of cardiovascular disease and potential contributing comorbidities critical.

Despite the widespread physiologic effects of hyperthyroidism, few studies have investigated its prevalence in patients with cardiovascular disease and its prognostic implications. Hyperthyroidism is significantly rarer than a cardiovascular disease with a prevalence of $1.2 \%$ in the US, but its physiologic effects are widespread and known to influence the cardiovascular system (4). Hyperthyroidism induces increased heart rate and myocardial contractility and is correlated with several forms of heart disease (5). Myocardial infarction (MI), ischemic stroke, and hemorrhagic stroke are among the most severe acute cardiovascular diseases, but their relationship with hyperthyroidism remains a subject of ongoing study. Several studies from Asia have found associations between these acute cardiovascular events and hyperthyroidism. Notably, a large Korean study found hyperthyroidism was independently associated with increased risk for $\mathrm{MI}$ and ischemic stroke but not for hemorrhagic stroke (6). In addition, a Taiwanese study found an independent association of hyperthyroidism with ischemic stroke in patients between 18 and 44 years of age (7). Our study will seek to build on prior studies by characterizing the relationship between hyperthyroidism and cardiovascular disease in a nationwide registry of US patients. We will attempt to identify the prevalence of thyrotoxicosis in patients presenting with cardiovascular events, determine the burden of MI and stroke in the presence of hyperthyroidism, and investigate the impact of hyperthyroidism on mortality. We present the following article in accordance with the STROBE reporting checklist (available at https://dx.doi.org/10.21037/gs-21-236).

\section{Methods}

\section{Data source}

This is a retrospective cohort study based on the Nationwide Readmission Database (NRD) from 2010 to 2017. NRD is part of the Healthcare Cost and Utilization Project (HCUP) covers $57.8 \%$ of the entire United States population, and is used to assess the cost and quality of healthcare services (8). The NRD database contains the following information: sociodemographic information, data about the diagnosis and procedures at hospitalization admission based on the International Classification of Diagnosis Ninth and Tenth Revision (ICD-9 and ICD-10) codes, and in-patient outcomes (Table S1). The study was conducted in accordance with the Declaration of Helsinki (as revised in 2013). Ethical review and approval from Tulane Institutional Review Board were waived for this study. Data was retrieved from NRD upon purchase through Dr. Kandil. Patient data was deidentified and individual consent for this retrospective analysis was waived.

\section{Study population}

Primary hospital admission records were screened for the diagnosis of newly diagnosed MI (codes: 410, I21, I22, I21A1), ischemic stroke (codes: 433, 434, I63), and hemorrhagic stroke (430-432, I600-I62). The generated sample was then surveyed for hyperthyroidism (codes: 242 and E05). Adult cohorts ( $\geq 18$ years) with and without hyperthyroidism were compared. Patients with a history of thyroiditis, hypothyroidism, previous MI, previous stroke, missing length of stay (LOS), or missing survival data were excluded.

\section{Study parameters}

Patient factors of interest included age (18-44, 45-64, 65+ years old), gender, median household income quartile for patient's zip code, and state of residence (in-state or out-ofstate). Comorbidities were identified based on Charlson's comorbidity index score (9). Risk factors for cardiovascular events were investigated, including smoking, hypertension, diabetes, hyperlipidemia, and body weight. The study also included the following characteristics: primary 
Table 1 Prevalence of hyperthyroidism in patients admitted for cardiovascular events

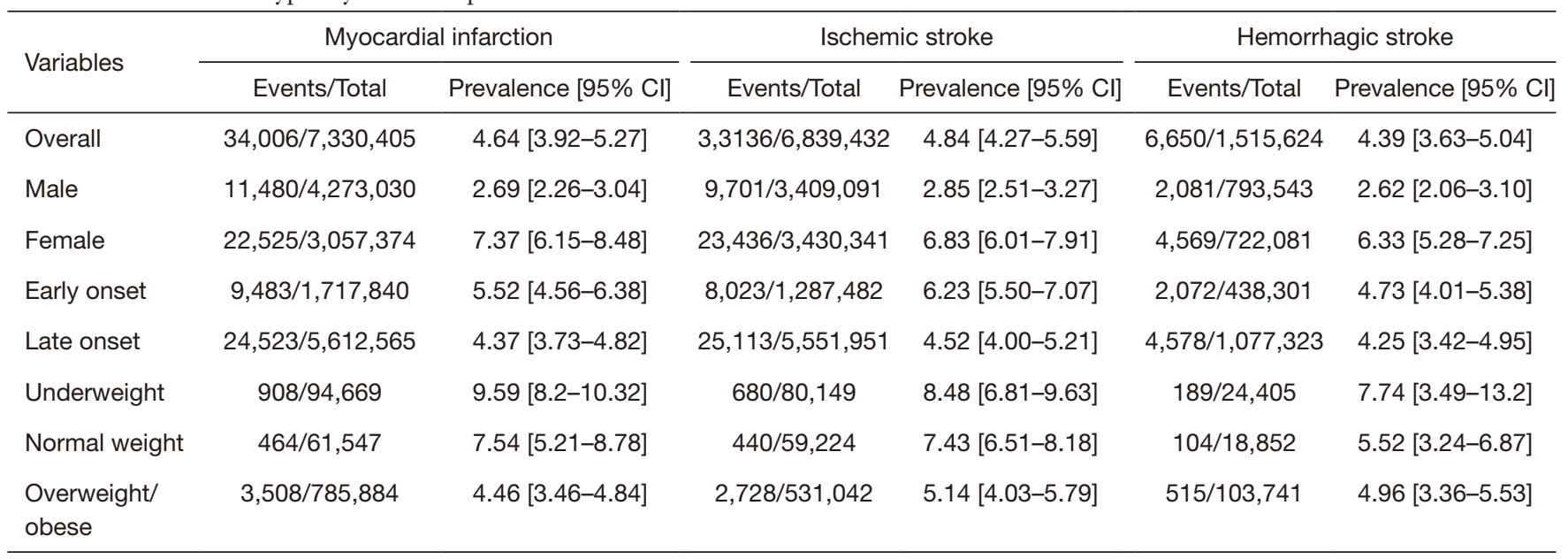

Annual prevalence of hyperthyroidism was calculated by dividing the number of prevalent cases in each year (i.e., those having hyperthyroidism-related claims) by the total number of patients admitted for newly diagnosed myocardial infarction, ischemic stroke, or hemorrhagic stroke. Stratification by sex, age at onset, and body weight revealed significant difference between subgroups (all $\mathrm{P}<0.001)$. $\mathrm{Cl}$, confidence interval.

insurance type (Medicare, Medicaid, private insurance, others), hospital teaching status (metropolitan teaching, metropolitan non-teaching, non-metropolitan), hospital bed size (small, medium, large), and discharge disposition (routine, transfer to short-term hospital, home health care, against medical advice). Outcomes of interest were inpatient mortality at primary admission, hospital LOS, and cost of health service (10).

The study objective was to (I) identify the prevalence of thyrotoxicosis in patients presenting with cardiovascular events in the United States, (II) determine the healthcare burden of MI and stroke in the presence of hyperthyroidism, and (III) investigate the impact of hyperthyroidism on mortality.

\section{Statistical analysis}

Data analysis used weighted measurements reflecting the national estimate level. All variables were categorized and presented as frequencies and percentages. Chi-square or Fisher's Exact tests were used to compare the categorical variables of the hyperthyroid cohorts and the control euthyroid group. For quantitative variables, data are presented as mean and standard deviation (SD) unless the normal assumption was violated, in which case median and interquartile range is shown. Unbiased and a priori cut-off values of quantitative variables were set at the median levels. Student's $t$-test and Mann-Whitney U tests were employed.
Prevalence of hyperthyroidism in newly diagnosed MI, ischemic stroke, and hemorrhagic stroke was calculated per 100,000 persons. The rates were further analyzed by sex, age, and body mass index. Binary logistic regression analysis was employed to identify the impact of hyperthyroidism on poor outcomes after adjustment for age and risk factors of cardiovascular events as smoking, hypertension, diabetes, hyperlipidemia, and body weight. Odds ratio (OR) and 95\% confidence interval (CI) were estimated. A significance level was set at $\mathrm{P}<0.05$. Statistical data analyses were carried out using SAS 9.4 for Windows (SAS Institute Inc., Cary, NC, USA) and SPSS version 27.0.

\section{Results}

Prevalence of hyperthyroidism in MI and stroke admissions

During the study period in question, the NRD contained $278,609,748$ total hospitalization records $(123,214,598$ males and 165,455,151 females), 7,330,405 of which were noted to have a new MI; $6,839,432$ had a new ischemic stroke; and 1,515,624 were diagnosed with a new hemorrhagic stroke (Figure S1).

Roughly $0.46 \%$ of MI admissions over the observed time frame had hyperthyroidism also present as a diagnosis ( $\mathrm{n}=34,006$; Table 1$) .0 .27 \%$ of men and $0.74 \%$ of women had concomitant hyperthyroidism. Patients with hyperthyroidism were significantly more likely to 
have early-onset MI compared to late-onset (5.5/1,000 vs. 4.4/1,000 cases, $\mathrm{P}<0.001)$. Hyperthyroidism was more likely to be concomitant in underweight patients than in normal or overweight/obese patients $(0.96 \%$ vs. $0.75 \%$ vs. $0.45 \%$, respectively, $\mathrm{P}<0.001$ ).

Overall, 4.8/1,000 patients with new-onset ischemic stroke were noted to have concurrent hyperthyroidism. Men with hyperthyroidism made up $0.29 \%$ and women $0.68 \%$ of the patient population. Early-onset ischemic stroke was more likely than late-onset for hyperthyroid patients $(6.2 / 1,000 v s .4 .5 / 1,000, \mathrm{P}<0.001)$. As with $\mathrm{MI}$, the prevalence of hyperthyroidism had an inverse relation with body weight (under $v s$. normal $v s$. over/obese: 8.5/1,000 vs. 7.4/1,000 vs. 5.1/1,000, respectively).

Turning to hemorrhagic stroke, hyperthyroid patients had a prevalence of 4.4/1,000 cases. Men comprised 2.6/1,000 and women 6.3/1,000 cases. Patients were statistically more likely to be younger at the onset with concomitant hyperthyroidism $(0.47 \%$ vs. $0.42 \%$, $\mathrm{P}<0.001)$. Underweight patients with hyperthyroidism accounted for 7.7/1,000 cases. Normal weight patients with hyperthyroidism had a prevalence of 5.5/1,000 cases and overweight/obese hyperthyroid patients had a prevalence of 5.0/1,000. Figure S2 shows the annual prevalence of hyperthyroid cases per 1,000 .

\section{Characteristics of the study population}

\section{MI}

Hyperthyroid patients were more likely to develop acute MI at an early age $(27.9 \%$ vs. $23.4 \%, \mathrm{P}<0.001$; Table 2$)$. They were more likely to be male $(66.2 \%$ vs. $41.6 \%, \mathrm{P}<0.001)$ and underweight or normal weight $(18.6 \%$ vs. $10.0 \%$ and $9.5 \%$ vs. $6.5 \%$, respectively, $\mathrm{P}<0.001$ ). Hyperthyroid patients that were diagnosed with new-onset $\mathrm{MI}$ were more likely to be smokers ( $34 \%$ vs. $33.4 \%, \mathrm{P}<0.001)$ and live in the same state in which they received treatment $(94.1 \%$ vs. $93.5 \%, \mathrm{P}<0.001)$. Patients with hyperthyroidism and MI were more likely to be in the lowest quartile of income than the euthyroid cohort (32.9\% vs. 31.3\%, $\mathrm{P}<0.001)$. They had a higher proportion of diabetes mellitus $(39.8 \%$ vs. $38.6 \%$, $\mathrm{P}<0.001)$ and hypertension $(79.3 \%$ vs. $76.3 \%, \mathrm{P}<0.001)$, and less frequent hyperlipidemia $(54.2 \%$ vs. $57.0 \%, \mathrm{P}<0.001)$ than their euthyroid counterparts. Hyperthyroid patients with MI were more likely to be treated at a metropolitan teaching hospital than a metropolitan non-teaching or nonmetropolitan hospital (62.2\% vs. 57.6\%, 29.7\% vs. 33.4\%, and $8.0 \%$ vs. $9.0 \%$, respectively, $\mathrm{P}<0.001$ ).

\section{Ischemic stroke}

Of patients who had a newly diagnosed ischemic stroke, patients with hyperthyroidism were more likely to be younger $(24.2 \%$ vs. $18.8 \%, \mathrm{P}<0.001)$ and female $(70.7 \%$ vs. $50.1 \%, \mathrm{P}<0.001)$, and less likely to be overweight or obese $(70.9 \%$ vs. $79.3 \%, \mathrm{P}<0.001)$. Smoking status did not correlate with hyperthyroidism or ischemic stroke $(30.8 \%$ vs. $30.7 \%, \mathrm{P}=0.5)$. Hyperthyroid stroke patients were more likely to be in the top or bottom quartiles of income $(19.9 \%$ vs. $19.3 \%, 32.0 \%$ vs. $30.8 \%$, respectively, $\mathrm{P}<0.001)$ than they were to be in the middle two quartiles and to live in the same state as they received care $(95.0 \%$ vs. $94.1 \%$, $\mathrm{P}<0.001)$. Ischemic stroke patients with hyperthyroidism had higher prevalence of hypertension $(82.2 \%$ vs. $81.8 \%$, $\mathrm{P}=0.02)$, but less frequent diabetes mellitus $(64.3 \%$ vs. 63.3\%, $\mathrm{P}<0.001)$ and hyperlipidemia $(46.1 \%$ vs. $43.4 \%$, $\mathrm{P}<0.001)$. Hyperthyroid patients with ischemic stroke were more likely to be treated at a metropolitan teaching hospital than a non-metropolitan or non-teaching hospital (60.6\% vs. $56.3 \%, 8.9 \%$ vs. $10.0 \%$, and $30.5 \%$ vs. $33.7 \%$, respectively, $\mathrm{P}<0.001)$.

\section{Hemorrhagic stroke}

In the third subgroup of hemorrhagic stroke and hyperthyroidism, patients with hyperthyroidism were younger $(31.2 \%$ vs. $28.9 \%, \mathrm{P}<0.001)$ women $(68.7 \%$ vs. $47.6 \%, \mathrm{P}<0.001)$, not overweight or obese $(63.8 \%$ vs. $70.6 \%, \mathrm{P}<0.001)$ and smokers $(25.7 \%$ vs. $24.0 \%, \mathrm{P}=0.001)$. A higher proportion were living in the same state as the hospital they were treated in $(92.4 \%$ vs. $91.5 \%, \mathrm{P}=0.009)$ and were in the lowest or highest quartile of income (30.4\% vs. $29.5 \%, 22.2 \%$ vs. $21.3 \%$, respectively, $\mathrm{P}=0.32$ ). A hyperthyroid patient was less likely to be diabetic (71.4\% vs. $73.0 \%, \mathrm{P}=0.002)$ and more likely to have hyperlipidemia $(38.7 \%$ vs. $35.8 \%, \mathrm{P}<0.001)$ and hypertension $(78.9 \%$ vs. $74.6 \%, \mathrm{P}<0.001)$. This subgroup was more often treated at a metropolitan teaching hospital than at a non-teaching metropolitan or a non-metropolitan hospital $(75.5 \%$ vs. $73.3 \%, 20.3 \%$ vs. $22.5 \%, 4.2 \%$ vs. $4.2 \%, \mathrm{P}<0.001)$.

\section{Healthcare burden and in-hospital mortality}

\section{MI}

Patients with hyperthyroidism were less likely to die during primary admission $(8.0 \%$ vs. $9.6 \%, \mathrm{P}<0.001)$. However, they were associated with long hospital stays $(62.7 \%$ vs. $54.3 \%$, $\mathrm{P}<0.001)$. They were more likely to be on Medicare $(67.1 \%$ vs. $64.4 \%, \mathrm{P}<0.001)$ and Medicaid $(9.9 \%$ vs. $7.4 \%, \mathrm{P}<0.001)$ 


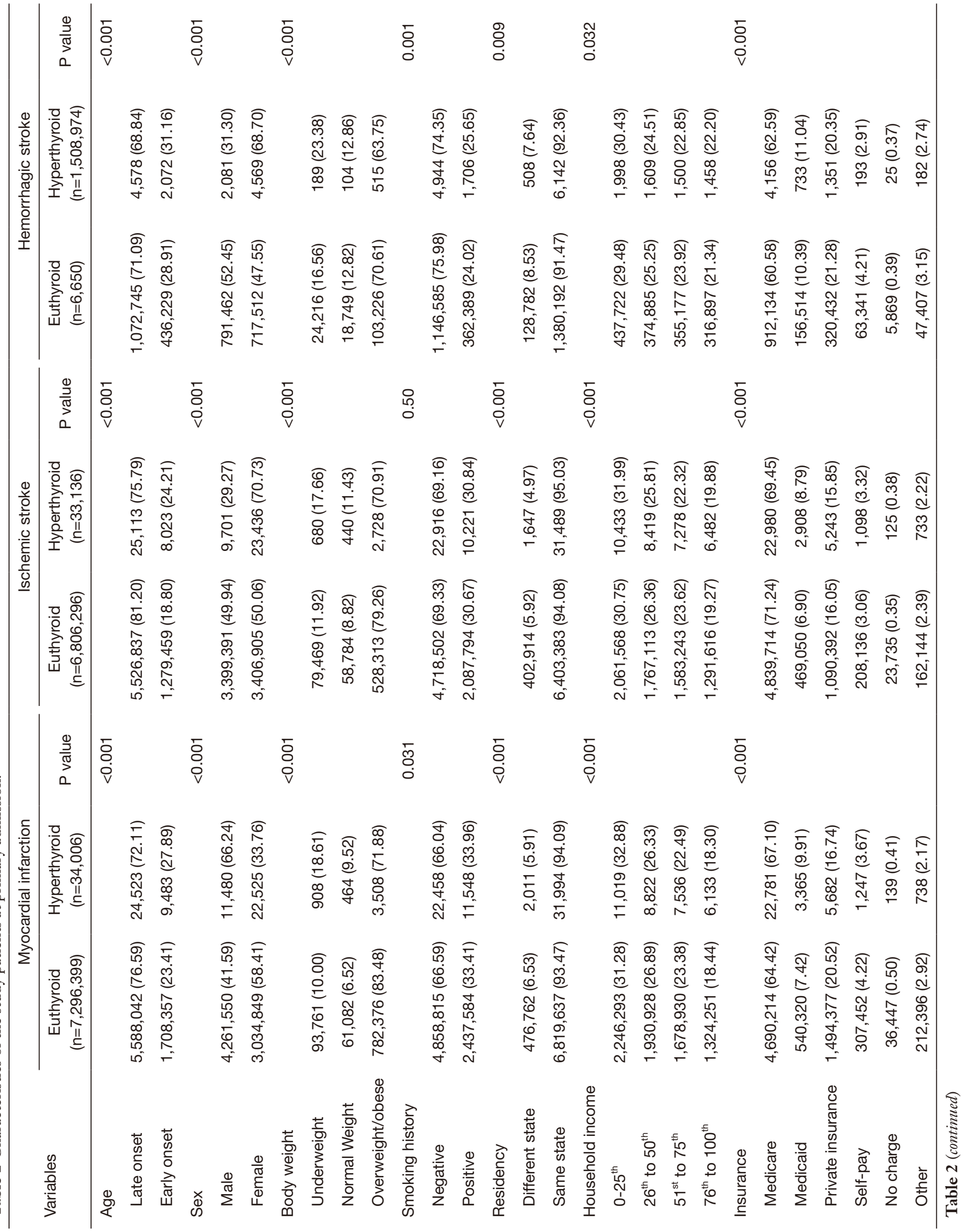




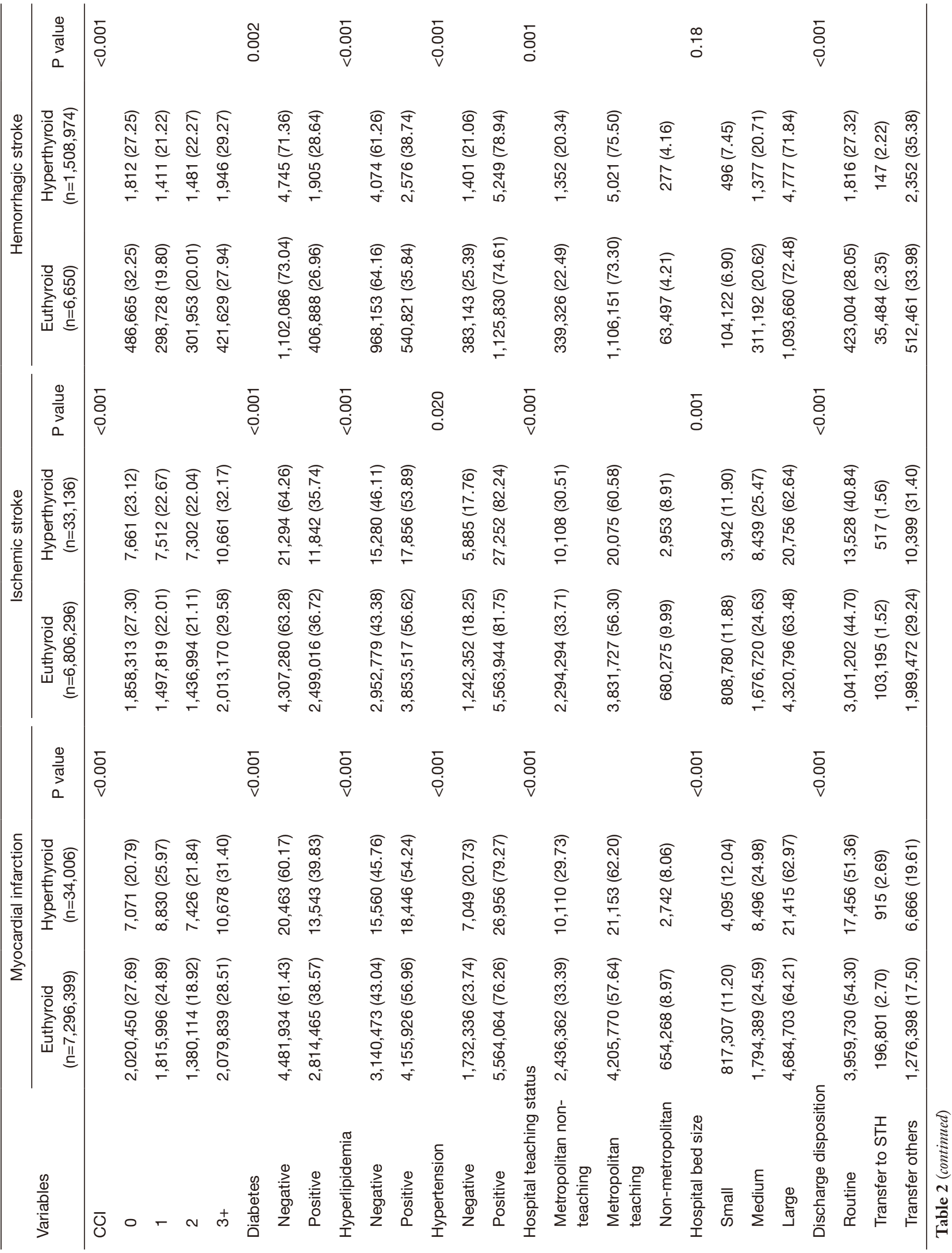




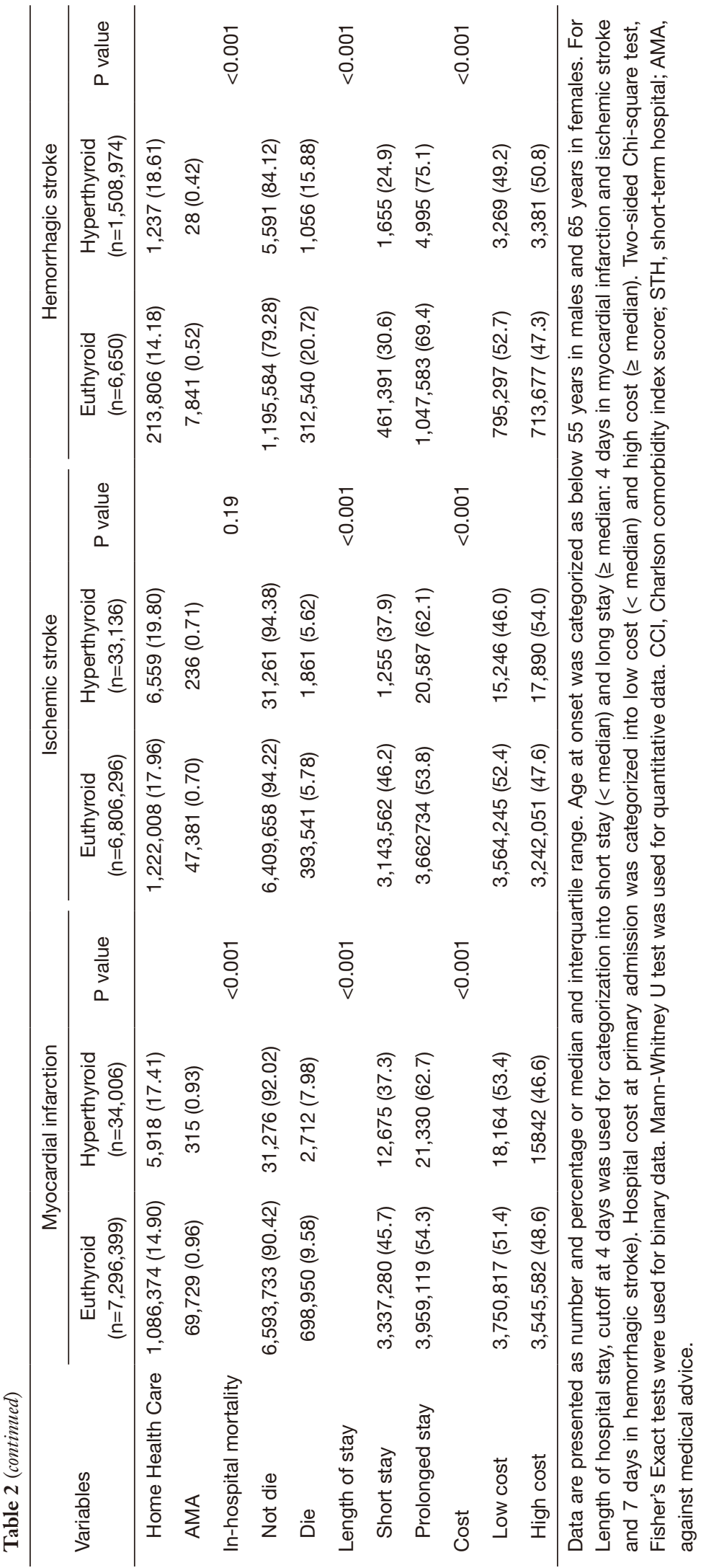


than private insurance $(16.7 \%$ vs. $20.5 \%, \mathrm{P}<0.001)$. Hyperthyroid patients were more likely to be admitted to a small hospital bed $(12 \%$ vs. $11.2 \%, \mathrm{P}<0.001)$ and discharged to home health care $(17.4 \%$ vs. $14.9 \%, \mathrm{P}<0.001)$ rather than routine discharge $(51.4 \%$ vs. $54.3 \%, \mathrm{P}<0.001)$.

\section{Ischemic stroke}

Ischemic stroke patients with concomitant hyperthyroidism had no difference in mortality $(5.6 \%$ vs. $5.7 \%, \mathrm{P}=0.19)$. However, they had a higher likelihood of longer duration of hospitalization $(62.1 \%$ vs. $53.8 \%, \mathrm{P}<0.001)$ and a high cost hospital stay $(54.0 \%$ vs. $47.6 \%, \mathrm{P}<0.001)$. They were less likely to be on Medicare $(69.5 \%$ vs. $71.2 \%, \mathrm{P}<0.001)$ or private insurance $(15.9 \%$ vs. $16.7 \%, \mathrm{P}<0.001)$, but more likely to be on Medicaid ( $8.8 \%$ vs. $6.9 \%, \mathrm{P}<0.001)$. They were more likely to have a medium sized bed $(25.5 \%$ vs. $24.6 \%, \mathrm{P}=0.001)$ and discharged to home health care $(19.8 \%$ vs. $18.0 \%, \mathrm{P}<0.001)$ rather than routine discharge $(40.8 \%$ vs. $44.7 \%, \mathrm{P}<0.001)$.

\section{Hemorrhagic stroke}

Patients with simultaneous hyperthyroidism and hemorrhagic stroke were more likely to die $(84.1 \%$ vs. $79.3 \%, \mathrm{P}<0.001)$, have a prolonged hospital stay $(75.1 \%$ vs. $69.4 \%, \mathrm{P}<0.001)$, and an above average cost of hospital stay (50.8\% vs. $47.3 \%, \mathrm{P}<0.001)$. They had a higher likelihood of being on Medicare $(62.6 \%$ vs. $60.6 \%, \mathrm{P}<0.001)$ and Medicaid $(11.0 \%$ vs. $10.4 \%, \mathrm{P}<0.001)$ than private insurance (20.4\% vs. $21.3 \%, \mathrm{P}<0.001$ ). Hyperthyroid hemorrhagic stroke patients were more likely to be discharged to home health care $(18.6 \%$ vs. $14.2 \%, \mathrm{P}<0.001)$ than routine discharge $(27.3 \%$ vs. $28.0 \%, \mathrm{P}<0.001)$.

\section{Factors influencing unfavorable outcomes}

Binary logistic regression was performed to determine individual risk factors for the three primary outcomes for these patients: mortality, prolonged stay, and high hospital cost.

\section{MI}

When stratifying patients that had a new onset diagnosis of MI, it was found that the risk of mortality increased with early age at onset (OR 2.33, 95\% CI: 2.31-2.35, $\mathrm{P}<0.001$ ), female sex (OR 1.21, 95\% CI: 1.20-1.21, P<0.001), and diabetes mellitus (OR 1.03, 95\% CI: 1.03-1.04, $\mathrm{P}<0.001$; Figure 1). Protective factors included positive smoking history (OR 0.62, 95\% CI: 0.62-0.63, P<0.001), hyperlipidemia (OR 0.43, 95\% CI: $0.43-0.43, \mathrm{P}<0.001$ ), hypertension (OR 0.68, 95\% CI: 0.68-0.69, $\mathrm{P}<0.001$ ), and thyrotoxicosis (OR 0.81, 95\% CI: 0.78-0.84, $\mathrm{P}<0.001$ ). Risk factors for a prolonged stay were early onset MI (OR 1.86, 95\% CI: $1.85-1.87, \mathrm{P}<0.001$ ), female sex (OR 1.34, 95\% CI: $1.34-1.35, \mathrm{P}<0.001$ ), diabetes (OR 1.49, $95 \%$ CI: $1.48-1.49, \mathrm{P}<0.001)$, hypertension (OR 1.12, 95\% CI: 1.11-1.12, $\mathrm{P}<0.001)$, and thyrotoxicosis (OR 1.35, 95\% CI: $1.32-1.38, \mathrm{P}<0.001)$. Positive smoking status (OR 0.84, 95\% CI: $0.83-0.84, \mathrm{P}<0.001)$ and hyperlipidemia (OR 0.66, 95\% CI: $0.66-0.67, \mathrm{P}<0.001)$ were associated with less risk of a prolonged hospital stay. Positive smoking status (OR 1.00, 95\% CI: $1.00-1.01, \mathrm{P}<0.001$ ), diabetes (OR 1.16, 95\% CI: $1.16-1.17, \mathrm{P}<0.001)$, and hyperlipidemia (OR $1.06,95 \%$ CI: $1.05-1.06, \mathrm{P}<0.001)$ were associated with high hospital costs. In contrast, early onset of MI (OR 0.85 , 95\% CI: 0.85-0.85, $\mathrm{P}<0.001$ ), female sex (OR 0.71, 95\% CI: $0.71-0.72, \mathrm{P}<0.001$ ), and hypertension (OR $0.90,95 \%$ CI: $0.89-0.90, \mathrm{P}<0.001)$ were associated with a lower risk of high hospital cost.

\section{Ischemic stroke}

Early age of onset for ischemic stroke is the single biggest risk factor for mortality, representing a $50 \%$ increase (OR 1.50, 95\% CI: $1.48-1.51, \mathrm{P}<0.001$ ), and female sex is associated with a $7 \%$ increase (OR $1.07,95 \%$ CI: 1.06-1.08, $\mathrm{P}<0.001$; Figure 2). All other measures identified were associated with a decreased risk of mortality: positive smoking history had a $38 \%$ decrease (OR 0.62, 95\% CI: 0.61-0.62, $\mathrm{P}<0.001$ ), diabetes had a $1 \%$ decrease (OR 0.99, 95\% CI: $0.98-0.99, \mathrm{P}<0.001)$, hyperlipidemia was associated with a $54 \%$ lower risk (OR $0.46,95 \%$ CI: $0.46-0.46$, $\mathrm{P}<0.001$ ), and hypertension had a $35 \%$ lower risk (OR 0.65, 95\% CI: $0.65-0.66, \mathrm{P}<0.001)$. Early onset age (OR 1.05, 95\% CI: $1.05-1.06, \mathrm{P}<0.001)$, female sex (OR 1.11, 95\% CI: $1.11-1.12, \mathrm{P}<0.001)$, diabetes history (OR 1.25, 95\% CI: $1.24-1.25, \mathrm{P}<0.001)$, hypertension (OR $1.08,95 \% \mathrm{CI}$ : $1.07-1.08, \mathrm{P}<0.001)$, and thyrotoxicosis (OR $1.38,95 \% \mathrm{CI}$ : 1.35-1.41, $\mathrm{P}<0.001)$ were all associated with an increased risk of prolonged hospital stay. In contrast, smoking history (OR 0.81, 95\% CI: 0.81-0.81, $\mathrm{P}<0.001$ ) and hyperlipidemia (OR 0.79, 95\% CI: $0.79-0.80, \mathrm{P}<0.001$ ) were associated with a lower risk of prolonged hospital stay. High hospital cost was more common in diabetic patients (OR 1.13, 95\% CI: $1.12-1.13, \mathrm{P}<0.001)$ and patients with thyrotoxicosis (OR 1.31, 95\% CI: 1.28-1.34, $\mathrm{P}<0.001$ ). Age (OR 0.83, 95\% CI: $0.83-0.84, \mathrm{P}<0.001$ ), female sex (OR 0.89, 95\% CI: 0.89-0.89, $\mathrm{P}<0.001$ ), positive smoking status (OR 0.96, 


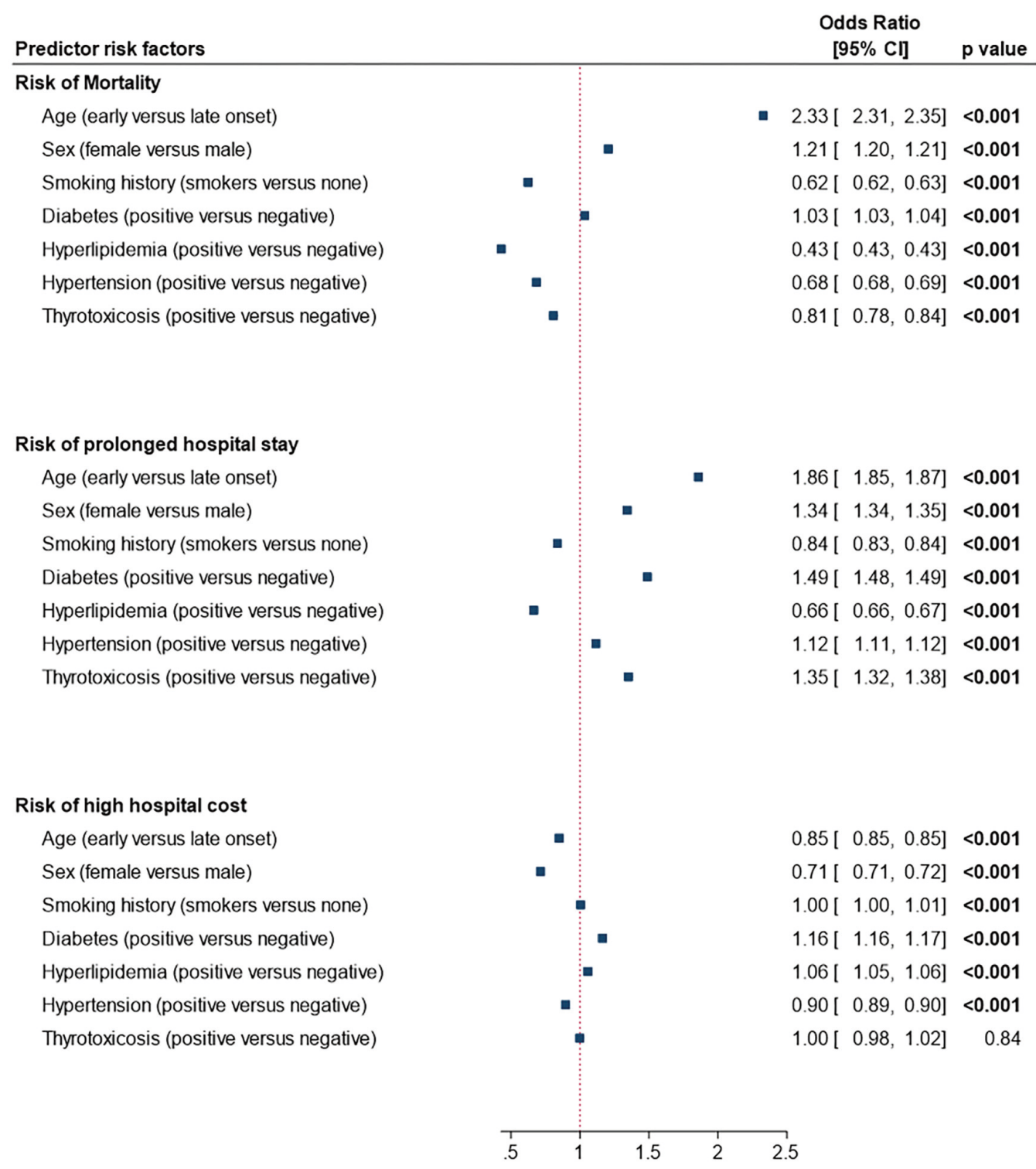

Figure 1 Predictors for poor outcomes in newly diagnosed myocardial infarction. Binary logistic regression analysis was performed. Results is reported as odds ratio and $95 \%$ confidence interval.

95\% CI: $0.95-0.96, \mathrm{P}<0.001)$, hyperlipidemia (OR 0.89, 95\% CI: 0.89-0.89, $\mathrm{P}<0.001)$, and hypertension (OR 0.96, 95\% CI: $0.95-0.96, \mathrm{P}<0.001)$ were all associated with lower risk of accruing a high hospital cost.

\section{Hemorrhagic stroke}

Increased risk of mortality was seen in early onset patients, who had a $47 \%$ increase (OR 1.47, 95\% CI: 1.46-1.49, $\mathrm{P}<0.001$ ), female patients had a $15 \%$ increase (OR 1.15 , $95 \%$ CI: $1.15-1.16, \mathrm{P}<0.001)$, and a $4 \%$ increase for patients with diabetes (OR 1.04, 95\% CI: 1.03-1.05, $\mathrm{P}<0.001$; Figure 3). Smoking was associated with a $23 \%$ decreased risk of mortality (OR $0.77,95 \%$ CI: $0.76-0.77$, $\mathrm{P}<0.001$ ), hyperlipidemia with a $34 \%$ decreased risk (OR $0.66,95 \%$ CI: $0.66-0.67, \mathrm{P}<0.001)$, hypertension with an $8 \%$ decreased risk (OR 0.92, 95\% CI: 0.91-0.93, P<0.001), and thyrotoxicosis was associated with a $28 \%$ decrease in mortality risk (OR $0.72,95 \%$ CI: $0.67-0.77, \mathrm{P}<0.001$ ). Risk of prolonged hospital stay was increased by $1 \%$ in smokers (OR 1.01, 95\% CI: $1.00-1.02, \mathrm{P}<0.001$ ), $18 \%$ in 


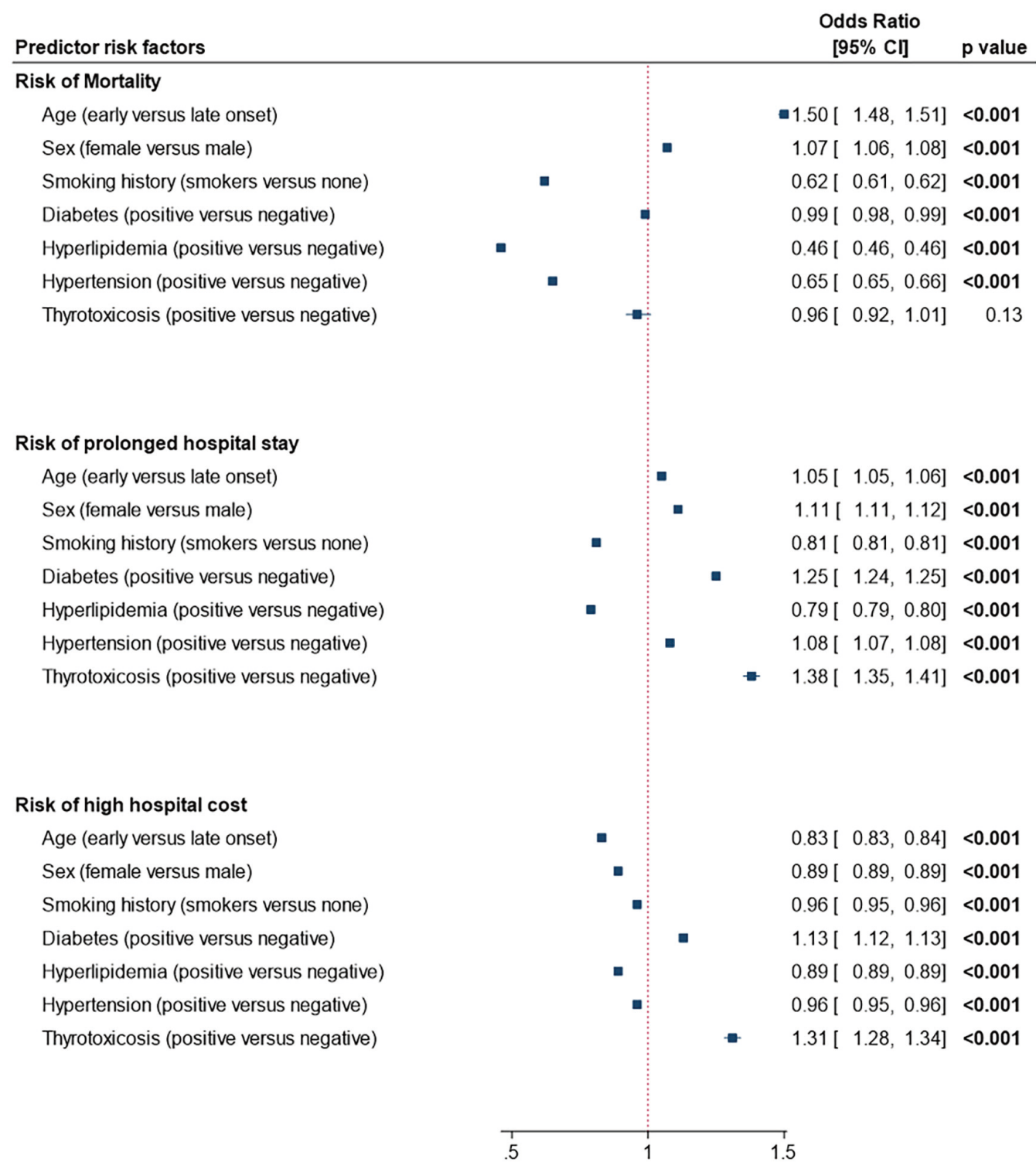

Figure 2 Predictors for poor outcomes in newly diagnosed ischemic stroke. Binary logistic regression analysis was performed. Results is reported as odds ratio and $95 \%$ confidence interval.

diabetics (OR 1.18, 95\% CI: $1.17-1.19, \mathrm{P}<0.001), 5 \%$ in hyperlipidemia (OR 1.05, 95\% CI: 1.04-1.06, P<0.001), $25 \%$ in patients with hypertension (OR $1.25,95 \%$ CI: $1.24-$ $1.26, \mathrm{P}<0.001)$, and $33 \%$ in patients with thyrotoxicosis (OR 1.33, 95\% CI: 1.25-1.40, $\mathrm{P}<0.001$ ). Early onset age of hemorrhagic stroke was found to be associated with a $31 \%$ decreased risk in prolonged hospital stay (OR 0.69, 95\% CI: $0.68-0.70, \mathrm{P}<0.001)$ and female sex was associated with an $8 \%$ decreased risk (OR 0.92, 95\% CI: 0.92-0.93,
$\mathrm{P}<0.001$ ). Diabetes (OR 1.10, 95\% CI: 1.09-1.11, $\mathrm{P}<0.001$ ), hypertension (OR 1.05, 95\% CI: 1.04-1.06, $\mathrm{P}<0.001$ ), and thyrotoxicosis (OR 1.18, 95\% CI: 1.12-1.24, $\mathrm{P}<0.001$ ) were all associated with increased risk of a high cost hospital bill. Conversely, early age of hemorrhagic stroke was associated with a $54 \%$ decreased risk of high cost (OR $0.46,95 \% \mathrm{CI}$ : $0.46-0.46, \mathrm{P}<0.001)$, as well as a $17 \%$ decreased risk for females (OR 0.83, 95\% CI: 0.82-0.83, P<0.001), and 4\% decrease for patients with hyperlipidemia (OR 0.96, 95\% 


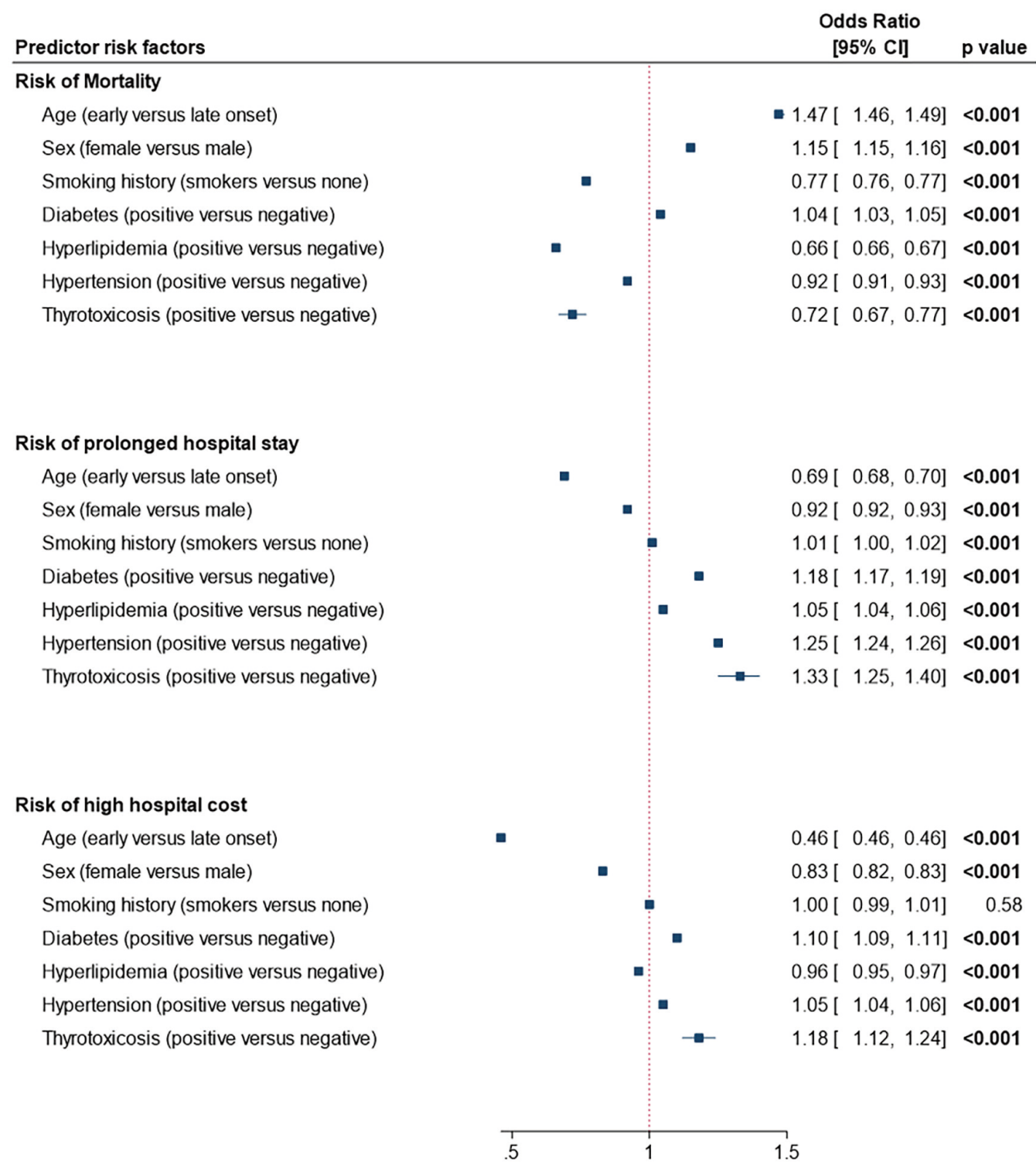

Figure 3 Predictors for poor outcomes in newly diagnosed hemorrhagic stroke. Binary logistic regression analysis was performed. Results is reported as odds ratio and $95 \%$ confidence interval.

CI: 0.95-0.97, $\mathrm{P}<0.001)$.

\section{Discussion}

Our retrospective cohort analysis from the NRD revealed concomitant hyperthyroidism in $0.46 \%$ of cases of MI, $0.48 \%$ of cases of ischemic stroke, and $0.44 \%$ of cases of hemorrhagic stroke. The prevalence of hyperthyroidism in patients presenting with $\mathrm{MI}$ and both subtypes of stroke is lower than the national prevalence for hyperthyroidism,
$1.2 \%$ (4). This raises the question of why hyperthyroidism is underrepresented in patients presenting for these conditions relative to its prevalence in the general population. Hypertension, high cholesterol, smoking, and obesity have been consistently shown to contribute to cardiovascular disease risk, including that of MI and stroke, and hyperthyroidism is associated with lower frequencies of obesity and hyperlipidemia and higher frequencies of hypertension and smoking (5,11-13). These characteristics were consistent with those of our study population. Thus, it 
remains unclear how the mixed risk profile of hyperthyroid patients contributes to their total cardiovascular disease risk. Our study shows a lower prevalence of concomitant hypertension in patients presenting for MI or stroke than would be expected given the basal prevalence of $1.2 \%$, which is a compelling counter to previous studies associating hyperthyroidism with increased risk for MI and stroke $(14,15)$. While the low overall prevalence of comorbid hyperthyroidism in patients presenting with $\mathrm{MI}$ and stroke is reassuring, this study saw a significantly higher proportion of early-onset MI and stroke in patients with hyperthyroidism. Early-onset ischemic stroke has previously been associated with hyperthyroidism, but other studies have not found the same risk in younger patients $(7,14)$. This raises an alarm that hyperthyroidism may play a role in the development of devasting cardiovascular events in younger patients.

Our investigation of mortality in patients with MI, ischemic stroke, and hemorrhagic stroke revealed a mixed picture of hyperthyroidism relative to mortality. Patients with concomitant hyperthyroidism presenting with $\mathrm{MI}$ and hemorrhagic stroke had lower mortality, while patients with ischemic stroke had no significant difference. Few studies have examined mortality in these cardiovascular events in patients with hyperthyroidism and none have shown a significant difference in mortality (6). Notably, younger patients and female patients were associated with increased mortality for all three conditions. This younger group of patients is the same group that had a higher correlation with comorbid hyperthyroidism at presentation. The role in which the hyperthyroidism variable impacts mortality risk is not readily apparent. In fact, hypertension, an adverse effect of hyperthyroidism, was associated with decreased mortality risk across all three conditions. One explanation could be that hyperthyroidism could lead to earlier presentation in younger, otherwise healthy patients. Another may be that the hyperdynamic state could confer unintended benefits in case of a cardiovascular event. Increased inotropy and chronotropy of the heart may increase the survivability of MI and stroke. The hyperdynamic state of the cardiac muscle in general may be sufficient to offset the damage and immobility of the heart wall in a younger patient with hyperthyroidism, leading to a higher chance of adequate compensation. When this effect is paired with a hypertensive state, it is possible that these two typically adverse conditions would be protective in the event of an MI or stroke. Further studies will need to be performed to elucidate possible mechanisms behind the impact of hyperthyroidism on the risk for mortality in each of these conditions.

This study demonstrated that hyperthyroidism increases the burden of health care and its associated cost. Patients with hyperthyroidism had significantly longer hospital stays than euthyroid patients. In addition, patients that presented with ischemic or hemorrhagic stroke and concomitant hyperthyroidism had significantly increased cost of care. The cost of care in patients presenting with MI and concomitant hyperthyroidism was higher than that of euthyroid patients but the difference was not significant. It makes intuitive sense that longer hospital stays would lead to a higher cost of care, so it is surprising that despite hyperthyroidism's association with a longer duration of hospitalization there was no significant increase in cost in MI patients. On further examination, the relationship is not so simple. Risk factors in MI patients for increased duration of stay, like female sex and age were negatively correlated with the cost of care. Parsing out the relationships and mechanisms of risk factors for increased cost of care in patients with MI or stroke remains a challenge. Whether the increases in LOS and cost are due to efforts to optimize care for a chronic disease before discharge or to treat acute complications related to cardiovascular disease is unknown. To compare with another chronic endocrine disorder, diabetes was associated with increased LOS and cost across all three conditions in our study. Thus, while our study does indicate that concomitant hyperthyroidism is positively associated with LOS across all conditions and cost of care in stroke patients, we are unable to implicate a specific cause for this relationship.

One of the major limitations of this study is some difficulty obtaining complete cumulative incidence and survival analysis in the patient cohort. The NRD does not trace patient outcomes between calendar years, so multiple patients were excluded from the study population due to database realities. Additionally, although the NRD is the largest patient care database available, it only contains data for roughly $60 \%$ of the US population, and all results are weighted estimates. It is possible, though unlikely, that some hospitals and patients not included in the NRD care for and consist of patients with different and unrepresented backgrounds and outcomes compared to the NRD. Another limitation is that as this study is purely retrospective cohort. Further studies will need to be performed in a prospective fashion to determine the underlying causes of the findings described above. Lastly, we found a positive relationship between hyperthyroidism, LOS, and cost of care in stroke 
patients; however this study is unable to determine the specific causes of the LOS and increased cost, and can only note that the correlation exists.

\section{Conclusions}

Patients that have experienced MI or hemorrhagic stroke with concomitant thyrotoxicosis were observed to have a lower mortality rate than euthyroid patients, but these conditions were also associated with an increased LOS. Ischemic stroke patients with hyperthyroidism did not have a similar drop in mortality. Patients who had ischemic or hemorrhagic stroke with thyrotoxicosis had a significantly higher hospital cost, comparatively. The hyperdynamic cardiovascular state underlying hyperthyroidism may play a protective role in these adverse events, however further investigation into the associated real-time physiological changes will need to be performed.

\section{Acknowledgments}

A sincere thank you to Loula Burton from Tulane's Research Proposal Development Office for her diligent editing and proofreading of this paper.

Funding: This work was supported by "Carol Lavin Bernick Grant" from Tulane University, New Orleans to Eman Toraih (PI) 07/01/2020-06/31/2021, and by a pilot grant from Tulane University Health Sciences Center to Emad Kandil.

\section{Footnote}

Reporting Checklist: The authors have completed the STROBE reporting checklist. Available at https://dx.doi. org/10.21037/gs-21-236

Conflicts of Interest: All authors have completed the ICMJE uniform disclosure form (available at https://dx.doi. org/10.21037/gs-21-236). EK serves as an Editor-inChief of Gland Surgery. ET reports that authors received "Carol Lavin Bernick Grant" and "pilot Grant" from Tulane University. Part of the fund was used to purchase the Nationwide Readmission Database. The fund received did not have any influence on the selection, conduction, or publishing the article. EK reports that he used part of the fund to purchase the Nationwide Readmission Database, that was used in the current analysis. However, the funding agency did not have any influence on the selection, conduction, or publishing the article. The other authors have no conflicts of interest to declare.

Ethical Statement: The authors are accountable for all aspects of the work in ensuring that questions related to the accuracy or integrity of any part of the work are appropriately investigated and resolved. The study was conducted in accordance with the Declaration of Helsinki (as revised in 2013). Ethical review and approval from Tulane Institutional Review Board were waived for this study. Data was retrieved from Nationwide Readmission Database upon purchase through Dr. Kandil. Patient data was deidentified and individual consent for this retrospective analysis was waived.

Open Access Statement: This is an Open Access article distributed in accordance with the Creative Commons Attribution-NonCommercial-NoDerivs 4.0 International License (CC BY-NC-ND 4.0), which permits the noncommercial replication and distribution of the article with the strict proviso that no changes or edits are made and the original work is properly cited (including links to both the formal publication through the relevant DOI and the license). See: https://creativecommons.org/licenses/by-nc-nd/4.0/.

\section{References}

1. Benjamin EJ, Virani SS, Callaway CW, et al. Heart Disease and Stroke Statistics-2018 Update: A Report From the American Heart Association. Circulation 2018;137:e67-e492.

2. Heidenreich PA, Trogdon JG, Khavjou OA, et al. Forecasting the future of cardiovascular disease in the United States: a policy statement from the American Heart Association. Circulation 2011;123:933-44.

3. Mutter R, Ali MM. Factors associated with completion of alcohol detoxification in residential settings. J Subst Abuse Treat 2019;98:53-8.

4. Singer PA, Cooper DS, Levy EG, et al. Treatment guidelines for patients with hyperthyroidism and hypothyroidism. Standards of Care Committee, American Thyroid Association. JAMA 1995;273:808-12.

5. Jabbar A, Pingitore A, Pearce SH, et al. Thyroid hormones and cardiovascular disease. Nat Rev Cardiol 2017;14:39-55.

6. Kim HJ, Kang T, Kang MJ, et al. Incidence and Mortality of Myocardial Infarction and Stroke in Patients with Hyperthyroidism: A Nationwide Cohort Study in Korea. 
Thyroid 2020;30:955-65.

7. Sheu JJ, Kang JH, Lin HC, et al. Hyperthyroidism and risk of ischemic stroke in young adults: a 5-year follow-up study. Stroke 2010;41:961-6.

8. Al-Qurayshi Z, Walsh J, Owen S, et al. Outcomes of head and neck surgery in patients with a history of solid organ transplantation. Laryngoscope 2020;130:E89-97.

9. Setter NW, Peres ML, de Almeida BMM, et al. Charlson comorbidity index scores and in-hospital prognosis of patients with severe acute respiratory infections. Intern Med J 2020;50:691-7.

10. Attia AS, Elnahla A, Hussein MH, et al. Impact of psychiatric comorbidities on outcomes related to thyroid and parathyroid operations. Surgery 2021;169:209-19.

11. Benjamin EJ, Muntner P, Alonso A, et al. Heart Disease and Stroke Statistics-2019 Update: A Report From the American Heart Association. Circulation

Cite this article as: Hussein M, Toraih E, Reisner ASC, Miller P, Corsetti R, Kandil E. Prevalence and mortality of cardiovascular events in patients with hyperthyroidism: a nationwide cohort study in the United States. Gland Surg 2021;10(9):2608-2621. doi: 10.21037/gs-21-236 2019;139:e56-e528.

12. Osman F, Franklyn JA, Holder RL, et al. Cardiovascular manifestations of hyperthyroidism before and after antithyroid therapy: a matched case-control study. J Am Coll Cardiol 2007;49:71-81.

13. Asvold BO, Bjøro T, Nilsen TI, et al. Tobacco smoking and thyroid function: a population-based study. Arch Intern Med 2007;167:1428-32.

14. Bano A, Peeters RP, Kavousi M. Response by Bano et al to Letter Regarding Article, "Thyroid Function and the Risk of Atherosclerotic Cardiovascular Morbidity and Mortality: The Rotterdam Study". Circ Res 2018;122:e18.

15. Dekkers OM, Horváth-Puhó E, Cannegieter SC, et al. Acute cardiovascular events and all-cause mortality in patients with hyperthyroidism: a population-based cohort study. Eur J Endocrinol 2017;176:1-9. 


\section{Supplementary}

Table S1 International Classification of Diagnosis codes

\begin{tabular}{|c|c|c|}
\hline Variable & \multicolumn{2}{|c|}{ Diagnostic code } \\
\hline \multicolumn{3}{|l|}{ Index diagnosis } \\
\hline Myocardial infarction & 410 & $|21| 22,, \mid 21 A 1$ \\
\hline Ischemic stroke & 433,434 & 163 \\
\hline Hyperthyroidism & 242 & E05 \\
\hline \multicolumn{3}{|l|}{ Exclusion criteria } \\
\hline Thyroiditis & 245 & E06 \\
\hline Hypothyroidism & 244 & E02, E03, E890 \\
\hline Hypertension & 40 & 11 \\
\hline Diabetes & 249,250 & E08, E09, E10, E11, E13 \\
\hline Hyperlipidemia & 272 & E781-E784, E7841, E7849, E785, E786 \\
\hline Obesity & V8521-V8525, V8531-V8539, V8541-V8545 & Z6825-Z6839, Z684, Z6841-Z6845 \\
\hline
\end{tabular}

ICD-9-CM/ICD-10-CM; International Classification of Diseases, Ninth/Tenth Revision, Clinical Modifications.

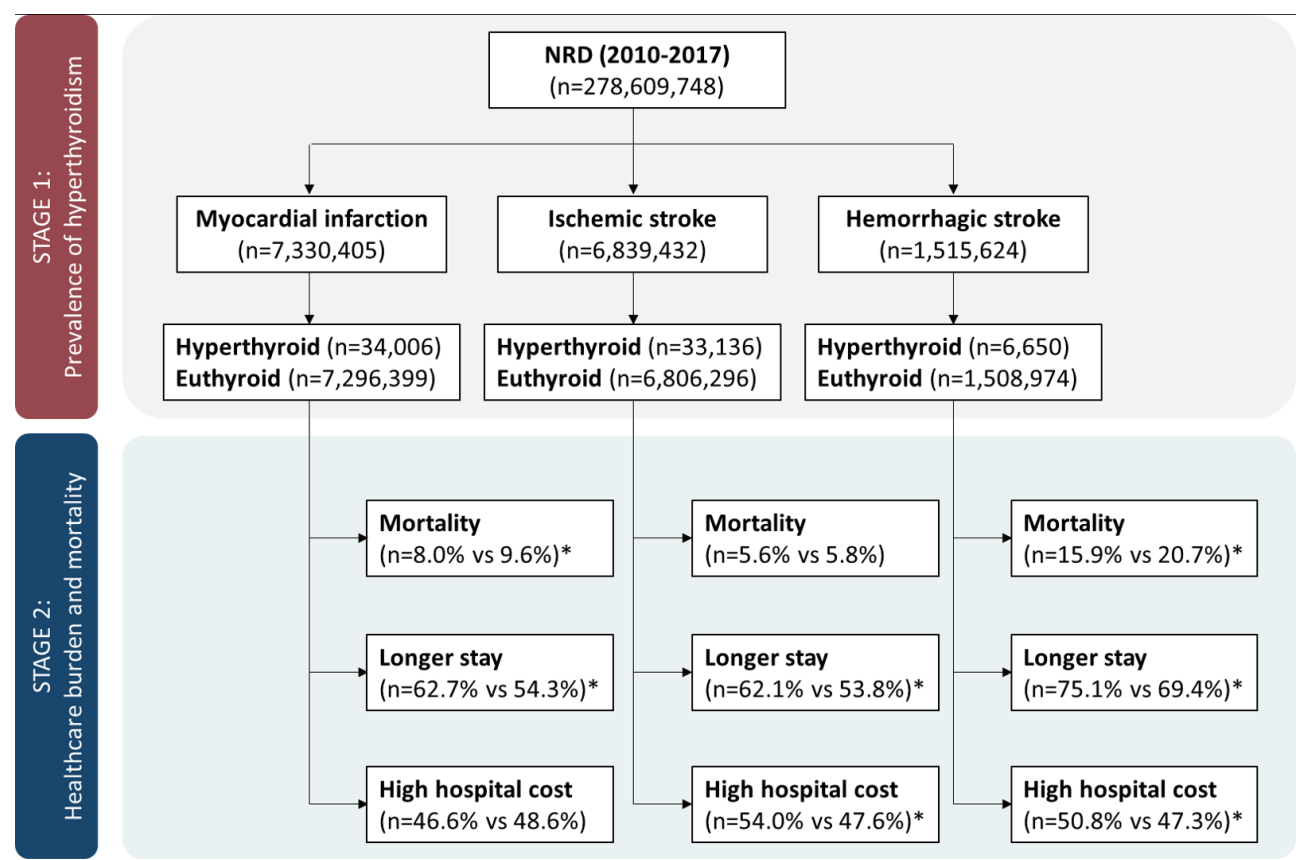

Figure S1 Graphical abstract. Selection process of patients admitted for newly diagnosed cardiovascular events. All values were weighted. *, $\mathrm{P}$ value is significant for comparison hyperthyroid versus euthyroid cohorts (all P value $<0.001$ ). Two-sided Chi-square test was used. 
(A)

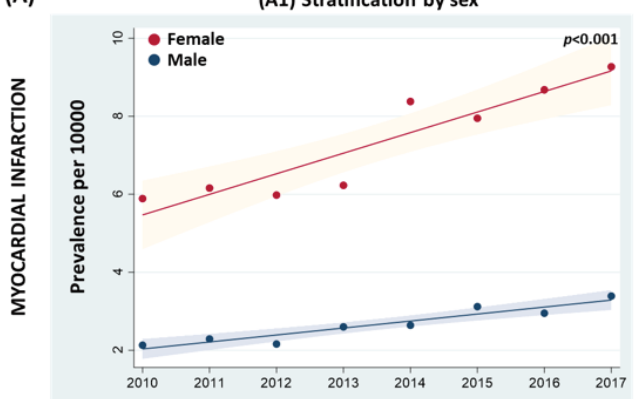

(B)

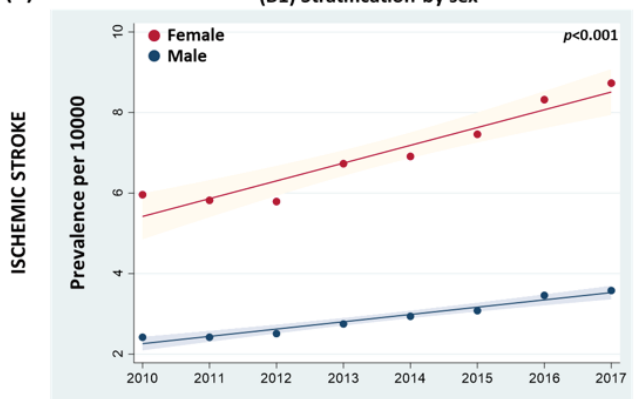

(C)

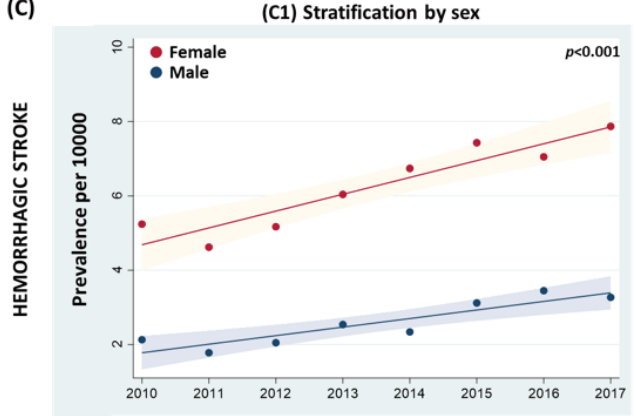

(A2) Stratification by age at onset

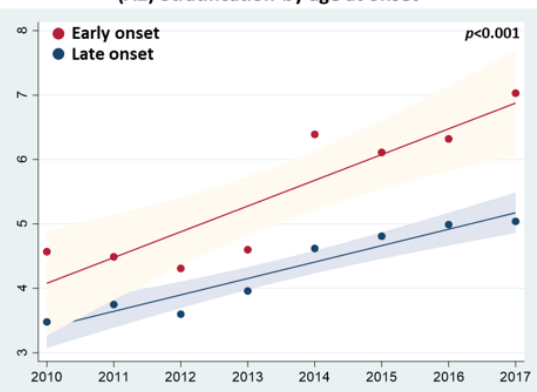

(B2) Stratification by age at onset

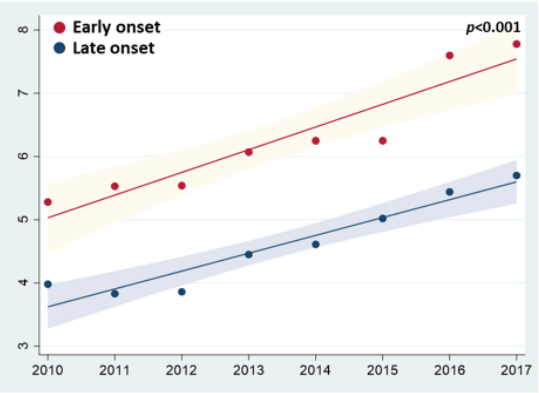

(C2) Stratification by age at onset

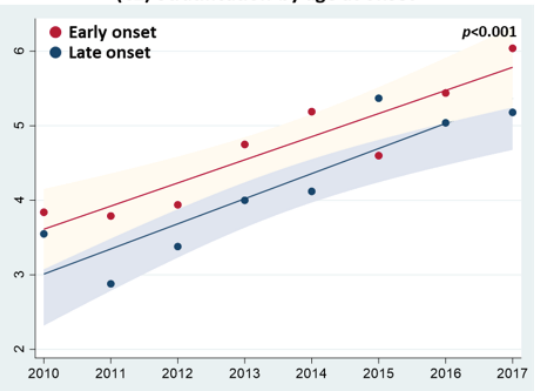

(A3) Stratification by Body weight

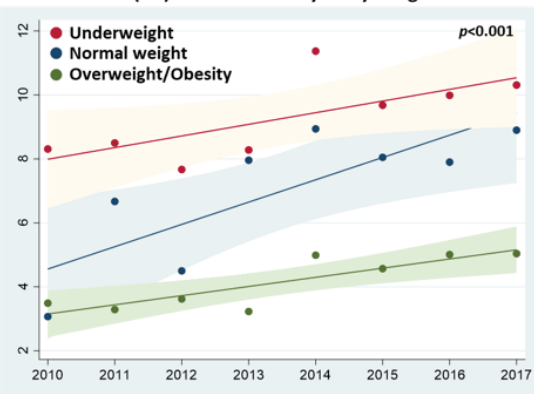

(B3) Stratification by Body weight

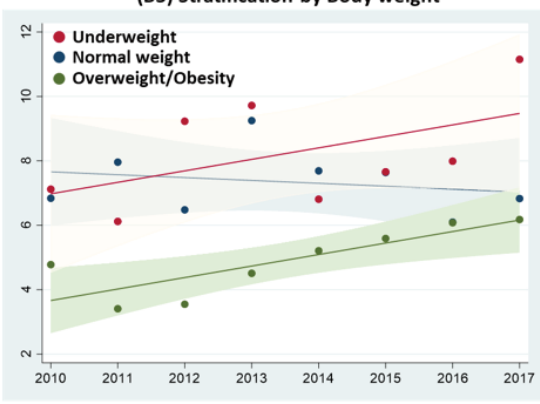

(C3) Stratification by Body weight

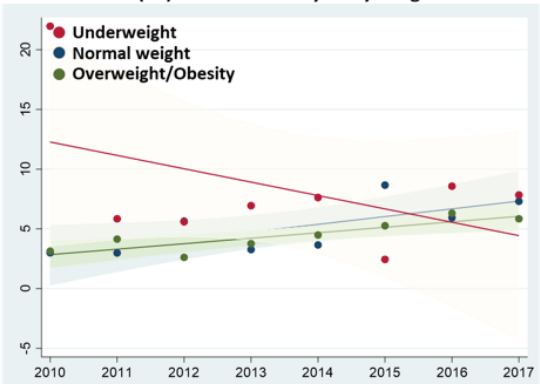

Figure S2 Annual prevalence of hyperthyroidism in newly diagnosed myocardial infarction and stroke stratified by sex, age, and body weight. Results are expressed as cases per 1000. Fitted line with 95\% confidence interval is shown. (A) Annual prevalence of hyperthyroidism in admitted patients for newly diagnosed myocardial infarction, stratified by sex, age, and body weight. Age at onset was stratified into early age at onset ( $<55$ years in males and $<65$ years in females) and late onset. Student's $\mathrm{t}$ and one-way ANOVA tests were used. All P values were $<0.001$. (B) Annual prevalence of hyperthyroidism in admitted patients for newly diagnosed ischemic stroke, stratified by sex, age, and body weight. Student's $\mathrm{t}$ and one-way ANOVA tests were used. All P values were $<0.001$ except in pairwise comparison between normal and underweight prevalence $(\mathrm{P}=0.25)$. (C) Annual prevalence of hyperthyroidism in admitted patients for newly diagnosed hemorrhagic stroke, stratified by sex, age, and body weight. In comparisons between male and female or early and late onset of disease, $\mathrm{P}$ values were $<0.001$. For body weight subgroup analysis, all pairwise comparisons were not significant (normal versus underweight: $\mathrm{P}=0.23$, normal versus overweight/obese: $\mathrm{P}=0.36$, and overweight/obese versus underweight: $\mathrm{P}=0.12$ ). 